\title{
Prescription of antidepressants to patients on opioid maintenance therapy - a pharmacoepidemiological study
}

\author{
Ingeborg Hartz ${ }^{1}$, Jørgen G. Bramness ${ }^{2,3}$ and Svetlana Skurtveit ${ }^{2,3}$ \\ 1) Department of Public Health Studies, Hedmark University College, Terningen Arena, NO-2400 Elverum, Norway \\ 2) Norwegian Centre for Addiction Research, University of Oslo, Norway \\ 3) Division of Epidemiology, Norwegian Institute of Public Health, Oslo, Norway
}

Korrespondanse: ingeborg.hartz@hihm.no

\begin{abstract}
Background and aims: Depression and anxiety are commonly reported among patients in opioid maintenance treatment (OMT). The aim of the present study was to describe aspects of prescription of antidepresant drug therapy among patients on OMT. Our research questions were: 1) What is the prevalence of antidepressant use according to age and gender? 2) Which antidepressants are used? 3) How are antidepressants used in terms of reimbursement codes, dispensed dose and duration of therapy?

Methods: Pharmacoepidemiological data were retrieved from the complete national Norwegian Prescription Database which contains information on all prescription drugs (such as Anatomical Theraputical Chemical (ATC)-code, Defined Daily Dose (DDDs)), dispensed at pharmacies to individual patients. Norwegian OMT-patients ( $\mathrm{N}=4374,3035$ men and 1339 women) who received methadone mixture, buprenorphine capsules or combined buprenorphine-naloxone capsules for at least 6 months in 2009 were included. Prevalence of antidepressant use in the studied patients was measured in terms of retrieval of prescriptions.

Results: During 2009 21.7\% of the studied patients filled at least one prescription for an antidepressant drugs (men: 21.2\%; women: 22.9\%). The subgroup of antidepressants most frequently dispensed was selective serotonin reuptake inhibitors (SSRIs) (33\%), followed by the sedative antidepressants mianserin and mirtazapin (22\%) and tricyclic antidepressants (TCAs) (20\%). Except for TCAs, prescriptions of all antidepressant subgroups were reimbursed for either anxiety or depression in $90 \%$ of the cases. Overall, $46.9 \%$ of the antidepressant users were prescribed antidepressants in the category $<1$ DDD per day and/or treatment $<3$ months, with no gender difference.

Conclusions: About one out of five OMT-patients filled a prescription for an antidepressant drug in 2009. Above $90 \%$ had their prescriptions reimbursed for either depression or anxiety. Use at low doses and/or sporadic use among half of the antidepressant users may reflect poor compliance or use for other maladies than the reimbursed disease code. Drug therapy for depression and anxiety among OMT-patients seems to be in line with recommendations for use; the SSRIs constitute the recommended drugs of choice due to their better effect/side effect profile, compared to the older antidepressants such as the TCAs.
\end{abstract}

\section{INTRODUCTION}

Depression is one of our most common psychiatric disorders with an estimated 12 month prevalence of $7-10 \%$ of the adult population ${ }^{1,2}$. Among drug and alcohol abusing individuals the prevalence rates are reported to be as high as $25-50 \%$. Prevalence rates of major depression among opiate-dependent patients enrolled in treatment programs range between $10-30 \%$ in international studies $^{5}$. This relatively large variation in prevalence may be explained by different study designs such as a varying length of participation in opioid maintenance (OMT)-programs at the time of diagnosis. Thus, depression and substance-dependencies commonly co-occur. This comorbidity has been associated with worse prognosis, poorer treatment outcomes, and increased risk of suicide $^{6-8}$.

OMT is the most common treatment for opiate addiction in Europe ${ }^{9}$. In Norway there are approximately 6000 patients in $\mathrm{OMT}^{10}$. A survey of Norwegian OMT patients in 2009 indicated an approximate prevalence rate of depressive symptoms of $16 \%$, and about $20 \%$ had an anxiety disorder or symptoms that could point to this ${ }^{10}$. There were, however, large regional variations in the prevalence rates, e.g. for depression ranging from $7 \%$ in the county of Buskerud to $30 \%$ among OMT-patients in the county of Hedmark. These estimates must be interpreted with great caution; information was missing in $8 \%$ of the patients, and the results were not based on the use of clinically validated questionnaires. Still, it isn't reasonable that the prevalence of depressive disorders in OMT patients should vary to this extent across the country.

According to data from the Norwegian Prescription Database (NorPD), 9.3\% of all adult women and 5.0\% of all men in Norway had at least one prescription of an antidepressant drug dispensed in 2004 and prevalence increased with age ${ }^{11}$. These figures resemble to a high degree the rates of depression in the population ${ }^{1,2}$. Antidepressants, however, are used also for several other conditions like anxiety disorders, eating disorder, pain management and to induce sleep. Thus, the preva- 
lence figures indicate that not all episodes of major depression are treated with antidepressants.

The pattern of antidepressant drug use in the general population is characterized by both an increasing use by age, and a higher level of use among women compared to men. To our knowledge, there is limited information on antidepressant drug therapy among patients enrolled in OMT-programs. Based on information from the nationwide NorPD, the aim of our study was to describe aspects of prescription of antidepressant drug therapy among patients on opioid maintenance therapy.

Our research questions were:

1. What is the prevalence of antidepressant use according to age and gender?

2. Which antidepressants are used?

3. How are antidepressants used in terms of reimbursement codes, dispensed dose (defined daily doses) and duration of therapy?

\section{MATERIAL AND METHODS}

\section{Data source}

Data were drawn from the NorPD ${ }^{12}$. From January $1^{\text {st }}$ 2004, all pharmacies in Norway have been obliged by law to submit data electronically to the NorPD on all dispensed prescriptions. The NorPD contains information on all prescription drugs, reimbursed or not, which are dispensed at pharmacies to individual patients outside institutions. Each record contains a unique person-identifier, which makes it possible to follow all prescriptions chronologically for each individual patient. Data on the prescribers are also collected, and each physician is identified by a unique identification code. Data on both patients and physicians are anonymized. Because data are collected from pharmacies, only prescribed drugs that are actually dispensed are captured in the NorPD. The data collected for our study were patients unique identifying number (encrypted), sex, age, the date of dispensing, and drug information (Anatomical Therapeutic Chemical (ATC)-code, Defined Daily Dose (DDDs)), and information on reimbursement codes. Reimbursement codes are wide categories of diseases, according to ICD-10 or ICPC coding, linking the prescribing to reimbursement. The following reimbursement codes were used: anxiety and depression: ICD code F3, F4 and ICPC code P73, P74, P76, P79, -74, -74, pain: ICD code -71, ICPC code -71 , and other indications: ICD code -70 .

All medicines dispensed in Norway are classified according to the ATC-classification system ${ }^{13}$. Quantities of dispensed drugs are measured in terms of the number of defined daily doses (DDDs). The basic definition of a DDD is the assumed average maintenance dose per day for a drug used for its main indication in adults $^{13}$.The DDD for each drug is determined by the WHO collaborating centre for drug statistics ${ }^{13}$. See Table 1 for all antidepressant substances included in this study with assigned defined daily doses (DDDs).

\section{OMT in Norway}

The Norwegian OMT model was implemented during 1998. To qualify for entry to the program, heroin users should have been dependent on heroin for several years and have had previous contact with abstinenceoriented treatment. OMT has only been available through a public funded program run by specialized centers ${ }^{14}$. According to the National Directorate of Health 4900 patients were included in OMT programs in Norway by the end of $2008^{15}$. Generally, OMT is provided as high-dose medication in Norway, and mean dosages for OMT medications in 2008 constituted $106 \mathrm{mg} /$ day for methadone and $18 \mathrm{mg} /$ day for buprenorphine ${ }^{16}$.

\section{Study population}

The study population was defined from retrieval of prescriptions of drugs used in OMT-programs during 2009. Due to relatively high numbers of patients entering and leaving OMT programs each year, our study population were restricted to include patients on OMT for six months or longer only. It has been reported that most patients who quit OMT, quit within the first year $^{17}$.

The study was based on prescription data from January $1^{\text {st }} 2009$ to December $31^{\text {st }} 2009$. Figure 1 presents a flow chart of the study population, with inclusion and exclusion of patients. To be included in the study patients had to meet the following criteria: being dispensed either methadone mixture (ATC code N07BC02), buprenorphine capsules (Subutex ${ }^{\circledR}$;

Table 1. Antidepressant substances registered in Norway in 2009, and included in the study, with assigned defined daily doses (DDDs).

\begin{tabular}{llr}
\hline ATC code & Name & $1 \mathrm{DDD}$ \\
\hline Tricyclic antidepressants & (TCAs) & \\
N06AA04 & clomipramine & $100 \mathrm{mg}$ \\
N06AA06 & trimipramine & $150 \mathrm{mg}$ \\
N06AA09 & amitriptyline & $75 \mathrm{mg}$ \\
N06AA10 & nortriptyline & $30 \mathrm{mg}$ \\
N06AA12 & doxepin & $100 \mathrm{mg}$ \\
Selective serotonin reuptake inhibitors (SSRIs) \\
N06AB03 & fluoxetine & $20 \mathrm{mg}$ \\
N06AB04 & citalopram & $20 \mathrm{mg}$ \\
N06AB05 & paroxetine & $20 \mathrm{mg}$ \\
N06AB06 & sertraline & $50 \mathrm{mg}$ \\
N06AB08 & fluvoxamine & $100 \mathrm{mg}$ \\
N06AB10 & escitalopram & $10 \mathrm{mg}$ \\
Sedative antidepressants & & \\
N06AX03 & mianserin & $60 \mathrm{mg}$ \\
N06AX11 & mirtazepin & $30 \mathrm{mg}$ \\
Others & & \\
N06AG02 & moclobemide & $300 \mathrm{mg}$ \\
N06AX12 & bupropion & $300 \mathrm{mg}$ \\
N06AX16 & venlafaxine & $100 \mathrm{mg}$ \\
N06AX18 & reboxetine & $8 \mathrm{mg}$ \\
N06AX21 & duloxetine & $60 \mathrm{mg}$ \\
\hline
\end{tabular}




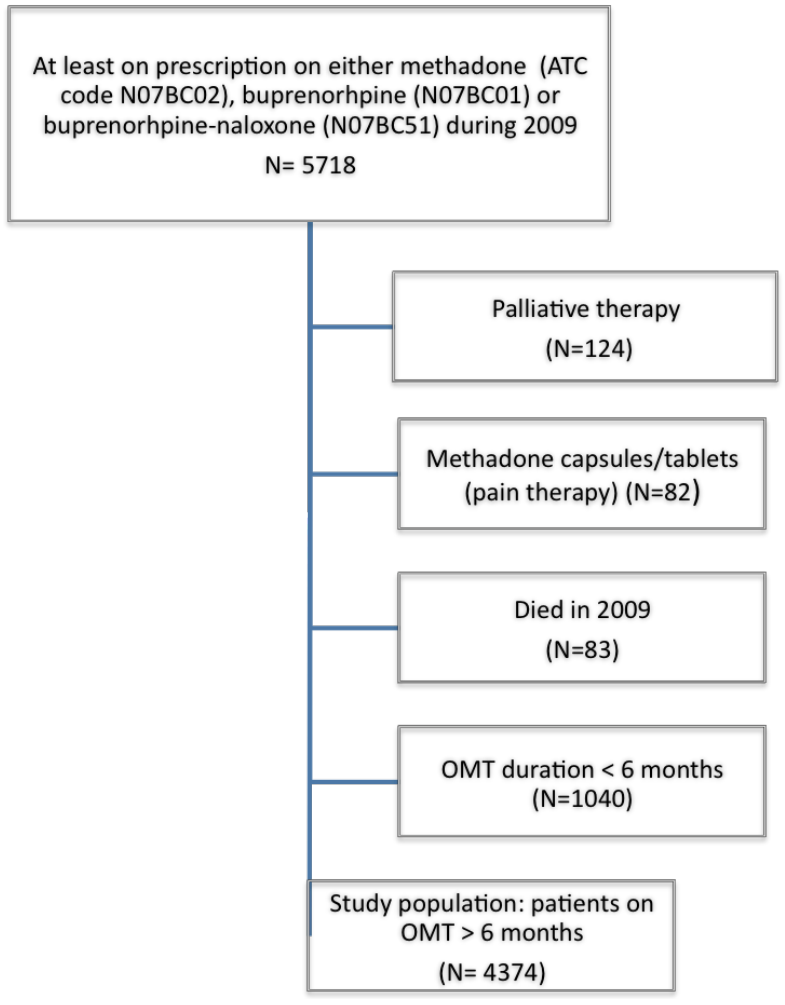

Figure 1. Flow chart of the study population, with inclusion and exclusion of patients. OMT $=$ Opioid maintenance therapy.

Table 2. Characteristics of Norwegian patients in opioid maintenance therapy (OMT) more than six months in 2009 according to age and type of therapy.

\begin{tabular}{lc}
\hline & OMT-patients \\
\hline Study population, N & 4374 \\
Men, n (\%) & $3035(69.4)$ \\
Age (mean, SD) & 41.6 (SD 8.0) \\
Methadone, n (\%) & $2489(56.9)$ \\
Buprenorphine, n (\%) & $1603(36.6)$ \\
Buprenorphine-naloxone combination; n (\%) & $819(18.7)$ \\
Two or more opioids*, n (\%) & $517(11.8)$ \\
\hline
\end{tabular}

*Patients who received two or more different opioids for maintenance therapy during the year.

N07BC01) or buprenorphine-naloxone combined capsules (Subuxone ${ }^{\circledR}$; N07BC51) during the study period. Patients who had been dispensed methadone mixture with the reimbursement code for palliative treatment of malignant disease or methadone capsules were not included in the study. Patients who were dispensed tablets or capsules of methadone were excluded because these formulations of methadone are in Norway primarily used in pain therapy. Patients who died during 2009 and patients who received opioid maintenance therapy for a short time (less than 6 months) were excluded from the study.
Patients who received their OMT-drug in specialized centers and institutions during the study period were not available for inclusion in our study because their OMT drugs dispensed in institutions are not captured by NorPD.

\section{Antidepressants available in Norway}

In Norway antidepressants are available as prescription drugs only. Registered antidepressants in Norway were categorized into 4 groups: 1) selective serotonin reuptake inhibitors (ATC code N06AB) 2) tricyclic antidepressants (N06AA) 3) sedative antidepressants; mianserin (N06AX03) and mirtazapin (N06AX11) and 4) others.

\section{Analyses and statistics}

One-year prevalences of antidepressant use is presented and defined as retrieval of at least one prescription of an antidepressant dug during 2009. Distribution of antidepressant subgroups and reimbursement codes among antidepressant users in 2009 are presented as proportions. Further, the amount of antidepressant drugs dispensed (DDDs per day) was calculated as follows: Total DDDs dispensed in 2009 divided by (the number of days between last and first OMT drug dispensed + number of DDDs at the last dispensed prescription of antidepressant). Aspects of antidepressant use according to dispensed dose (DDDs) per day and duration of therapy were explored using the following four categories:

$>1 \mathrm{DDD} /$ day and treatment $>3$ months

$>1 \mathrm{DDD} /$ day and treatment $<3$ months

$<1 \mathrm{DDD} /$ day and treatment $>3$ months

$<1 \mathrm{DDD} /$ day and treatment $<3$ months

The software SPSS 17.0 for Windows was applied for data management and descriptive statistics.

\section{RESUlts}

\section{Study population}

4374 patients were included in the study, of whom $69 \%$ were men (table 2). Overall, mean age was 41.6 (SD 8.0) years and median age was 42 years, and the men were on average older than the women. More than half of the study population received methadone for opioid maintenance therapy, whereas the remaining patients received either buprenorhpine or buprenorhpine-naloxone in combination (no gender difference) (table 2).

\section{Prevalence of antidepressants dispensed to patients on $\mathrm{OMT}$}

During 2009, 21.7\% (949 out of 4374) of the study population filled at least one prescription for an antidepressant drug (men: 21.2\%; women: 22.9\%). Figure 2 shows prevalences of antidepressant drug use by age. The highest prevalence of antidepressant use was ob- 


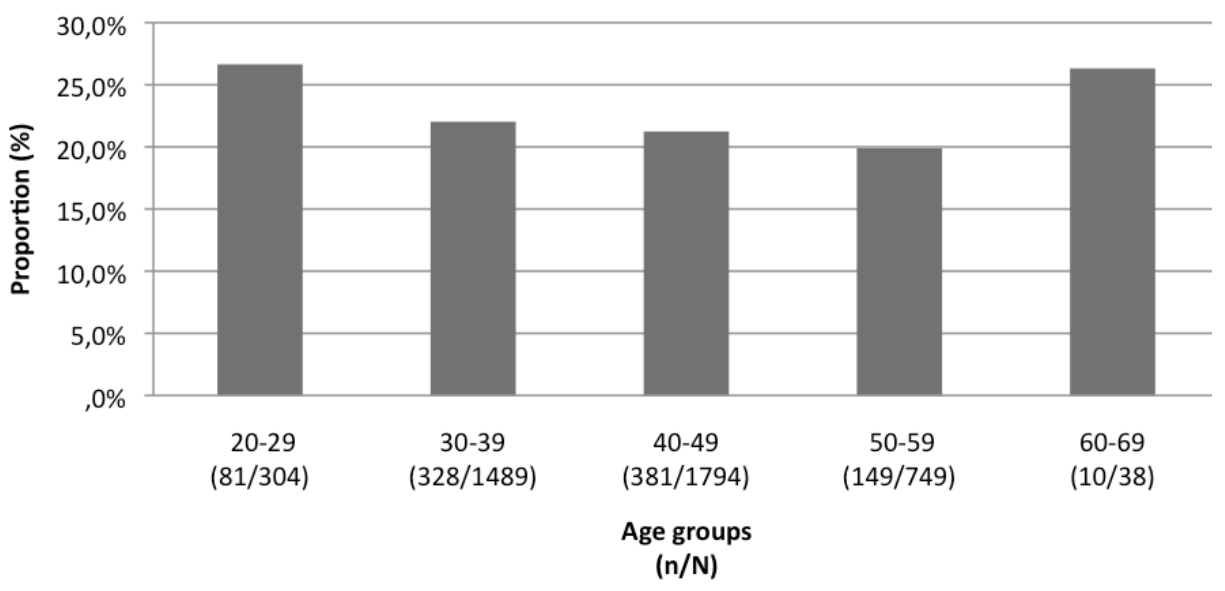

Figure 2. Proportion of patients on opioid maintenance therapy who had at least one antidepressant drug dispensed in 2009, according to age. Percent.
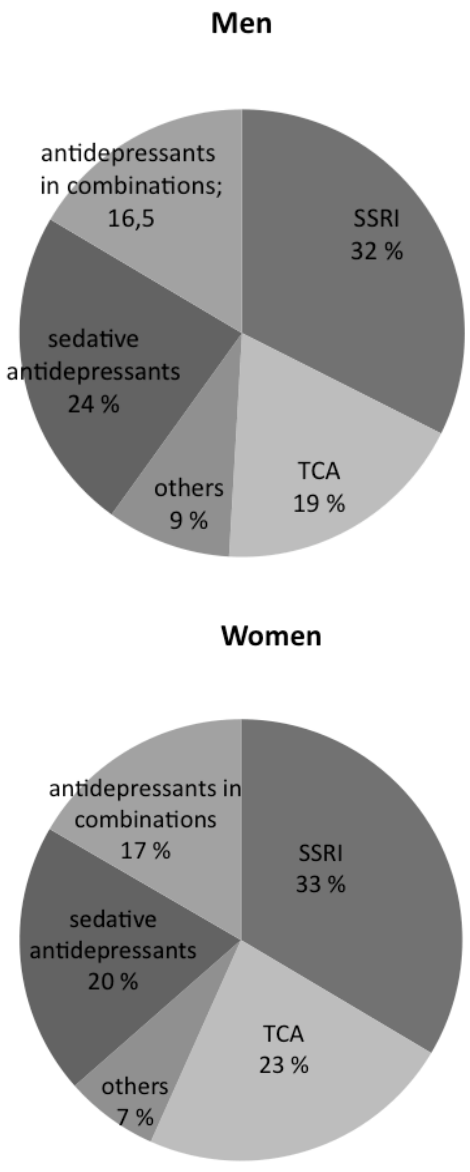

Figure 3. Distribution of antidepressant subgroups among patients on opioid maintenance treatment who had at least one antidepressant drug dispensed in 2009. Percent.

served among the youngest, aged 20-29 years, and oldest OMT patients, aged 60-69 years.

Figure 3 shows choice of antidepressant subgroups among OMT patients who had dispensed at least one prescription of antidepressant drug in 2009. The subgroup of antidepressants most frequently dispensed was SSRI (33\%), followed by the sedative antidepressants mianserin and mirtazapin (22\%), TCA (20\%), antide- pressants in combination during the year (17\%) and others $(8 \%)$. A similar pattern of use was observed among men and women (figure 3 ).

\section{Antidepressants use according to reimbursement codes, dispensed dose and duration of therapy}

Table 3 shows antidepressant use according to reimbursements codes. Prescriptions of all antidepressant subgroups were reimbursed for either anxiety or depression in $90 \%$ of the cases, except TCAs in which about three out of four of all prescriptions were reimbursed for for anxiety or depression (men: $74.6 \%$, women: $76.1 \%$ ).

Table 4 shows antidepressant use according to doses per day and duration of treatment. Overall, $46.9 \%$ of the antidepressant users were prescribed antidepressants in the category $<1$ DDD per day and/or treatment $<3$ months. This was similar in both genders (men 46.7\%; women 47.2\%). Among TCA users, $92.6 \%$ of the patients were prescribed antidepressants at doses $<1$ DDD per day and/or treatment $<3$ months.

\section{Discussion}

Overall; $22 \%$ of Norwegian OMT patients were on antidepressant drug therapy, in which about $90 \%$ were reimbursed for either depression or anxiety disorders. According to annual assessments of all OMT-centers in Norway in $2009,16 \%$ of all OMT-patients had symptoms of depression, and about $20 \%$ had an anxiety disorder or symptoms that could point to this ${ }^{10}$. However, we do not know the extent to which these symptoms are overlapping in these patients. Thus, the present study cannot conclude on a divergence between the prevalence depression and anxiety morbidity and the drug treatment among OMT-patients.

Interestingly, approximately half of the antidepressant drug users were prescribed drugs at low daily doses (less than a DDD per day) or over a short period of time. These aspects of antidepressant drug use among OMT-patients could have several explanations. First, 
Table 3. Distribution of antidepressants according to reimbursement code among patients on opioid maintenance treatment in 2009. Number and percent.

\begin{tabular}{lrcccc}
\hline Reimbursement code & & $\begin{array}{c}\text { depression/ } \\
\text { anxiety }\end{array}$ & pain & other & $\begin{array}{c}\text { without reim- } \\
\text { bursement code }\end{array}$ \\
\hline Men & $\mathbf{N ~ ( \% )}$ & $\mathbf{n ~ ( \% )}$ & $\mathbf{n ~ ( \% )}$ & $\mathbf{n ~ ( \% )}$ & $\mathbf{n}(\mathbf{\%})$ \\
\cline { 2 - 6 } SSRI* $^{*}$ & $208(100)$ & $200(96.2)$ & 0 & $1(0.4)$ & $8(3.9)$ \\
TCA** & $119(100)$ & $90(75.6)$ & $4(3.4)$ & 0 & $25(21.0)$ \\
new sedative antidepressants & $151(100)$ & $138(91.4)$ & 0 & 0 & $13(8.6)$ \\
others & $58(100)$ & $52(89.7)$ & $1(1.7)$ & 0 & $6(10.3)$ \\
$\quad$ combinations & $106(100)$ & $103(97.2)$ & $1(0.9)$ & 0 & $3(2.8)$ \\
Women & & & & & \\
SSRI* & $103(100)$ & $102(99.0)$ & 0 & 0 & $1(1.0)$ \\
TCA** & $71(100)$ & $54(76.1)$ & $4(5.6)$ & 0 & $13(18.3)$ \\
new sedative antidepressants & $61(100)$ & $56(91.8)$ & 0 & 0 & $5(8.2)$ \\
others & $21(100)$ & $21(100)$ & $1(4.8)$ & 0 & 0 \\
combinations & $51(100)$ & $50(98.0)$ & $4(7.8)$ & 0 & $1(2.0)$ \\
\hline
\end{tabular}

* SSRI- selective serotonin reuptake inhibitors

** TCA- tricyclic antidepressants

Table 4. Distribution of antidepressants according to daily doses and duration of treatment among patients on opioid maintenance treatment in 2009. Number and percent.

\begin{tabular}{|c|c|c|c|c|c|}
\hline & $\begin{array}{c}\text { Use of } \\
\text { antidepressants }\end{array}$ & $\begin{array}{c}\text { doses }<1 \mathrm{DDD} / \text { day } \\
\text { and treatment } \\
<3 \text { months }\end{array}$ & $\begin{array}{c}\text { doses }>1 \mathrm{DDD} / \text { day } \\
\text { and treatment } \\
<3 \text { months }\end{array}$ & $\begin{array}{c}\text { doses }<1 \mathrm{DDD} / \text { day } \\
\text { and treatment } \\
>3 \text { months }\end{array}$ & $\begin{array}{c}\text { doses }>1 \mathrm{DDD} / \text { day } \\
\text { and treatment } \\
>3 \text { months }\end{array}$ \\
\hline Men & N (\%) & n (\%) & n (\%) & n (\%) & n (\%) \\
\hline SSRI* & $208(100)$ & 0 & $21(10.1)$ & $34(16.3)$ & $153(73.6)$ \\
\hline $\mathrm{TCA}^{* *}$ & $119(100)$ & $12(10.1)$ & $43(36.1)$ & $58(48.7)$ & $6(5.0)$ \\
\hline sedative antidepressants & $151(100)$ & $5(3.3)$ & $39(25.8)$ & $26(17.2)$ & $81(53.6)$ \\
\hline others & $58(100)$ & $2(3.4)$ & $15(25.9)$ & $9(15.5)$ & $32(55.2)$ \\
\hline antidepressants in combination & $106(100)$ & 0 & $6(5.7)$ & $30(28.3)$ & $70(66.0)$ \\
\hline \multicolumn{6}{|l|}{ Women } \\
\hline SSRI* & $103(100)$ & $1(1.0)$ & $8(7.8)$ & $22(21.4)$ & $71(69.9)$ \\
\hline TCA** & $71(100)$ & $3(4.2)$ & $28(39.4)$ & $32(45.1)$ & $8(11.3)$ \\
\hline sedative antidepressants & $61(100)$ & $2(3.3)$ & $18(29.5)$ & $10(16.4)$ & $31(50.8)$ \\
\hline others & $21(100)$ & 0 & $5(23.8)$ & $6(28.6)$ & $10(47.6)$ \\
\hline antidepressants in combination & $51(100)$ & 0 & $3(5.9)$ & $7(13.7)$ & $41(80.4)$ \\
\hline
\end{tabular}

* SSRI- selective serotonin reuptake inhibitors

** TCA- tricyclic antidepressants

antidepressants may also be used at lower doses to alleviate sleep problems or as adjunctive therapy for pain. Even if more than $90 \%$ of the prescriptions were reimbursed for depression or anxiety disorders, we know from earlier observations that reimbursement (disease) codes that trigger reimbursement are used to help the patient, rather than being exact codes for the disease at hand. Thus the relatively high frequency use of low doses and/or more sporadic use may reflect use for other maladies than indicated by the reimbursement code. Second, in addition to diagnosis, prescribed antidepressant drug dose depends on patients' age and body weight, and treatment duration depends on therapeutic efficacy and adverse side effects, which all constitute rational reasons for some patients to be prescribed lower daily doses than a DDD per day and/or stop therapy. Third, poor compliance with prescribed anti- depressant therapy may also contribute to patterns of sporadic retrieval of antidepressant drug prescriptions observed in this study. In this context, although prescribed antidepressants may be dispensed, prescription data do not reveal any information on the extent to which the dispensed drugs actually are ingested.

Even though there is evidence that depression among opiate-dependent patients has been associated with worse prognosis, there is a debate on how to treat OMT-patients with concurrent depression. Firstly, studies of antidepressant drug therapy for depression during OMT have shown varied results ${ }^{18}$. A recent review concluded that there is mixed and possibly poor evidence supporting the clinical use of antidepressants for the treatment of depression in opioid addicts that are treated with opioid agonists ${ }^{19}$. It is not clear what accounts for the differences in response to anti- 
depressant drug therapy, and more research is needed to determine how to select opiate-dependent patients most likely to benefit from antidepressants.

Secondly, the debate concerns the complex relationship between depression and opioid dependence. Depression may be a primary disorder, or may be secondary to the toxic and withdrawal effects of drugs, or of psychosocial stress associated with addiction ${ }^{18,20}$. Entry into OMT-programs, and reduction of drug use, has in several studies been associated with improvement in depressive symptoms in many OMT-patients with major depression ${ }^{18,20}$. However, not all patients will have such a favorable response to entry into OMTprograms. Our study population included OMTpatients on long term stable OMT (6 months or longer), most likely belonging to the latter group.

Among the OMT-patients in our study SSRIs constituted the antidepressant drug of choice. Only one out of five of the antidepressant users were prescribed TCAs, of whom about a quarter had their TCAs reimbursed for other indications than depression or anxiety. Further, only $5-10 \%$ of all TCA users had TCAs dispensed at daily doses over a duration of time indicative of use for depression of anxiety, compared to $70 \%$ of all SSRI users. Thus, this observation may reflect that TCAs, when prescribed, probably mainly are used for other problems than anxiety or depression. A reasonable conclusion from this may be that drug therapy for depression and anxiety among OMT-patients seems to be in line with recommendations for use; the SSRIs constitute the recommended drugs of choice due to their better effect/side effect profile, compared to the older antidepressants such as the TCAs.

Patterns of use of antidepressant drugs in the general population in Norway is characterized by both an increasing use by age, and a higher level of use among women compared to men ${ }^{11}$. According to the NorPD, $7.9 \%$ of all women and $4.2 \%$ of all men in Norway had at least one prescription of an antidepressant drug dispensed in $2009^{12}$. Further, 4.1\% of all 20-29 year olds had at least one prescription of an antidepressant drug dispensed in 2009 , increasing to $9.6 \%$ of all 60 69 year olds. Compared to the general population, a higher proportion of OMT-patients were dispensed antidepressants, with no gender difference, or an increasing use by age. A higher level of use may be as expected, and in agreement with the higher prevalence of depression and anxiety (and other maladies) among OMT-patients compared to the general population. Similar level of use irrespective of age and gender among OMT-patients, may also reflect different patterns of morbidity among OMT-patients compared to the general population.

The major strength of this study is that all dispensed drugs, both opioid maintenance drugs and antidepressants are captured at an individual level, and that each individual can be followed over time with data on all dispensed drugs. On the other hand, as mentioned, there may be several limitations using information from NorPD as a datasource to describe patterns of drug use in the population; the dispensed drugs may not necessarily be consumed, the reimbursement code may not reflect the true reason for prescribing a drug, and the DDD may deviate from the actual prescribed daily dose.

In conclusion, about one out of five long-term OMT-patients had an antidepressant drug dispensed in 2009 , of whom above $90 \%$ had their prescriptions reimbursed for either depression or anxiety. Use at low daily doses and/or sporadic use among half of the antidepressant users may reflect poor compliance or use for other maladies than the reimbursed disease code. Drug therapy for depression and anxiety among OMT-patients seems to be in line with recommendations for use; the SSRIs constitute the recommended drugs of choice due to their better effect/side effect profile, compared to the older antidepressants such as the TCAs.

\section{REFERENCES}

1. Kringlen E, editor. Psykiatri. Oslo: Gyldendal Akademisk, 2001.

2. Wittchen HU, Jacobi F, Rehm J, Gustavsson A, Svensson M, Jonsson B, et al. The size and burden of mental disorders and other disorders of the brain in Europe 2010. Eur Neuropsychopharmacol 2011; 21 (9): 655-79.

3. Landheim AS, Bakken K, Vaglum P. Gender differences in the prevalence of symptom disorders and personality disorders among poly-substance abusers and pure alcoholics. Substance abusers treated in two counties in Norway. Eur Addict Res 2003; 9 (1): 8-17.

4. Lyne JP, O'Donoghue B, Clancy M, O'Gara C. Comorbid psychiatric diagnoses among individuals presenting to an addiction treatment program for alcohol dependence. Subst Use Misuse 2011; 46 (4): 351-8.

5. Peles E, Schreiber S, Naumovsky Y, Adelson M. Depression in methadone maintenance treatment patients: rate and risk factors. $J$ Affect Disord 2007; 99 (1-3): 213-20.

6. Kosten TR, Rounsaville BJ, Kleber HD. A 2.5-year follow-up of depression, life crises, and treatment effects on abstinence among opioid addicts. Arch Gen Psychiatry 1986; 43 (8): 733-8.

7. Rounsaville BJ, Kosten TR, Weissman MM, Kleber HD. Prognostic significance of psychopathology in treated opiate addicts. A 2.5-year follow-up study. Arch Gen Psychiatry 1986; 43 (8): 739-45. 
8. Tondo L, Baldessarini RJ, Hennen J, Minnai GP, Salis P, Scamonatti L, et al. Suicide attempts in major affective disorder patients with comorbid substance use disorders. J Clin Psychiatry 1999; 60 Suppl 2: 63-9; discussion 75-6, 113-6.

9. European Monitor Centre for Drugs and Drug Addiction (EMCDDA). 2009 Annual report on the state of the drugs problem in Europe. Available from: http://www.emcdda.europa.eu/publications/annual-report/2009.

10. Waal H, Clausen T, Håseth A, Lillevold P. The 2009 Annual Assessment of the Norwegian OMT-programme. SERAF, Oslo, Norway. Report number 1/2010. Available at: http://www.med.uio.no/klinmed/forskning/ sentre/seraf/publikasjoner/rapporter/2010/SERAF-rapport-nr-1-2010-Statusrapport-2009-revidert.pdf.

11. Bramness JG, Hausken AM, Sakshaug S, Skurtveit S, Ronning M. [Prescription of selective serotonin reuptake inhibitors 1990-2004]. Tidsskr Nor Laegeforen 2005; 125 (18): 2470-3.

12. The Norwegian Prescription Database, National Institute of Public Health. http://www.norpd.no/.

13. Guidelines for ATC classification and DDD assignment. WHO Collaborating Centre for Drug Statistics Methodology, Norwegian Institute of Public Health, Oslo, Norway.

14. Waal H. Merits and problems in high-threshold methadone maintenance treatment. Evaluation of medicationassisted rehabilitation in Norway 1998-2004. Eur Addict Res 2007; 13 (2): 66-73.

15. Norwegian Directorate of Health, 2010. Nasjonal Retningslinje for Legemiddelassistert Rehabilitering ved Opiatavhengighet. Oslo, Norway, IS-1701.

16. Waal H, Clausen T, Haseth A, Lillevold P. The 2008 Annual Assessment of the Norwegian OMTprogramme. SERAF, Oslo, Norway. Report number 2/2009.

17. Simpson DD, Joe GW, Rowan-Szal GA. Drug abuse treatment retention and process effects on follow-up outcomes. Drug Alcohol Depend 1997; 47 (3): 227-35.

18. Nunes EV, Sullivan MA, Levin FR. Treatment of depression in patients with opiate dependence. Biol Psychiatry 2004; 56 (10): 793-802.

19. Pani PP, Vacca R, Trogu E, Amato L, Davoli M. Pharmacological treatment for depression during opioid agonist treatment for opioid dependence. Cochrane Database Syst Rev (9): CD008373.

20. Nunes EV, Levin FR. Treatment of depression in patients with alcohol or other drug dependence: a metaanalysis. JAMA 2004; 291 (15): 1887-96. 